\title{
On the association between rheumatoid arthritis and classical HLA class I and class II alleles predicted from single-nucleotide polymorphism data
}

\section{Mathieu Lemire}

Address: Ontario Institute for Cancer Research, 101 College Street, Suite 800, MaRS Centre, South Tower, Toronto, ON M5G0A3 Canada E-mail: mathieu.lemire@oicr.on.ca

from Genetic Analysis Workshop 16

St Louis, MO, USA 17-20 September 2009

Published: 15 December 2009

BMC Proceedings 2009, 3(Suppl 7):S33 doi: 10.1186/1753-656I-3-S7-S33

This article is available from: http://www.biomedcentral.com/I753-656I/3/S7/S33

(c) 2009 Lemire; licensee BioMed Central Ltd.

This is an open access article distributed under the terms of the Creative Commons Attribution License (http://creativecommons.org/licenses/by/2.0), which permits unrestricted use, distribution, and reproduction in any medium, provided the original work is properly cited.

\begin{abstract}
Using single-nucleotide polymorphisms (SNPs), we sought to predict classical class I and class II human leukocyte antigen (HLA) alleles, and test for their associations with rheumatoid arthritis (RA) in the North American Rheumatoid Arthritis Consortium sample of cases and controls, genotyped on the Illumina HumanHap550 BeadChip. We use publicly available databases of SNP data and HLA data to find SNPs or SNP-haplotypes to be used as surrogates for each HLA allele. To reduce the confounding effects of linkage disequilibrium with the HLA-DRBI locus, we tested for the association conditional on the presence or absence of a shared epitope allele on the same haplotype as the target HLA allele. Using SNP surrogates, we find that components of the DQ8 serotype (DQAI*030I:DQBI*0302) are associated with RA, irrespective of the presence or absence of a shared epitope allele on their respective haplotypes. Knowledge of the haplotype structure in the HLA region is still necessary for better interpretation of the results.
\end{abstract}

\section{Background}

The human leukocyte antigen (HLA)-DRB1 locus has long been recognized as a strong genetic risk factor for rheumatoid arthritis (RA), yet it explains less than half of the estimated genetic susceptibility to the disease [1]. Largescale studies that interrogate the whole genome have uncovered, at strict significance thresholds, genetic risk variants outside the major histocompatibility complex (MHC) [2-4], while also replicating known associations from candidate gene studies [5]. The difficulty in evaluating the role of other candidate loci from the MHC region in the etiology of the disease resides in the strong linkage disequilibrium (LD) and the extended haplotype structure that exists in this highly polymorphic portion of the genome. We seek to evaluate the risk of other HLA loci, by using SNP data and publicly available HLA data, in order to predict and evaluate the effect of classical class I and class II HLA alleles in the North American Rheumatoid Arthritis Consortium (NARAC) case-control dataset [3], as distributed for use at the Genetic Analysis Workshop 16. 


\section{Methods}

The NARAC sample consists of 868 cases of RA and 1194 controls, all genotyped on the Illumina HumanHap550 BeadChip, or equivalent. The sample is fully described by Plenge et al. [3]. The sample also includes HLA-DRB1 alleles, typed at various resolutions. The susceptibility alleles at DRB1 tend to share the RAA motif in position 72-74 of the amino acid sequence, an observation that led to the hypothesis of a functional unit, called the shared epitope (SE) [6]. Amino acids found in positions 70-71 provide further refinement of the classification of DRB1 risk alleles [7].

To predict classical HLA alleles from SNP data, we followed the methods described by de Bakker et al. [8]. They typed six class I and class II HLA genes (A, B, C, DQA1, DQB1, and DRB1) in a set of samples that includes the CEU samples from the HapMap (Utah residents with ancestry from northern and western Europe). Most of the HLA alleles they report are at a resolution of four digits. We used this publicly available dataset, combined with SNP genotype data from the HapMap that are in the broad MHC region (chr6: 25, 990, 507...33, 893, 423 [hg18]), and that overlap with the set of SNPs on the Illumina HumanHap550 BeadChip. Using the CEU HapMap data combined with the CEU HLA data from de Bakker et al. [8], we searched for tags for each of the HLA alleles, considering up to three-SNP haplotypes as potential predictors. The best predictor was chosen based on the largest observed $r^{2}$ measure of LD (where, for each target HLA allele, we merged all other alleles at that locus into a single one, to mimic a biallelic locus; the same for multiple SNP haplotypes). To be considered a potential predictor of HLA alleles, a SNP had to be in Hardy-Weinberg equilibrium $(p>0.00001)$ in the set of controls from the NARAC dataset, and had to have a call rate above 95\% over all samples. We used the program Tagger [9] as implemented in computer program Haploview [10] to predict the HLA alleles from the HapMap SNP data.

We tested for the association between RA and the class I and class II (non DRB1) HLA alleles, using the SNP predictors as surrogates. Because the DRB1 locus is a strong risk factor for RA, we reduced the confounding effects of LD by performing the analysis conditional on whether each of the two alleles found at the DRB1 locus are members of the SE class of alleles, considering this conditioning argument as if it was a biallelic locus. We used the computer program UNPHASED [11] to perform the conditional tests of association. For each target HLA allele, we report two conditional odds ratios (ORs): these are ORs for the target HLA allele given the presence (SE+) or absence (SE-) of an SE allele on its haplotype. Among the four-digit alleles that are classified as SE+ (according to the classification of du Montcel et al. [7]), those that were actually observed in the NARAC samples only include DRB1*0101, *0102, *0401, *0404, *0405, *0408, and * 1001 .

The NARAC sample is affected by population substructure, with chi-square statistics reported to be inflated on average by a factor $\sim 1.4$ [3]. To account for the hidden ancestry of all cases and controls, we computed the spectral decomposition of a covariance matrix between all DNA samples and used its eigenvectors as surrogates for ancestry [12]. The covariance matrix was calculated using a set of $\sim 120,000$ autosomal SNPs that are at most modestly correlated (pairwise $r^{2}<0.30$ ), a set that excludes SNPs on the short arm of chromosome 6 and on the short arm of chromosome 8 (for reasons explained by Plenge et al. [3]). As in Plenge et al. [3], we found seven outliers by inspecting the eigenvectors associated with the top 10 eigenvalues: their respective entries in at least one eigenvector differed from the mean by more than six standard deviations. We removed these seven outliers from any downstream analyses, and recomputed the eigenvectors. As in Plenge et al. [3], the top three vectors that are statistically significant predictors of case-control status were used as surrogates for the hidden ancestry of all samples, and were used to correct for the effects of population stratification. By using them as covariates in a logistic regression framework, the inflation factor of all association results, excluding results on the short arm of chromosome 6, was calculated to be 1.035 . This value is similar to what has been calculated by Plenge et al. [3]. We used these three vectors as potential confounders in UNPHASED.

\section{Results}

We only report the results of the conditional tests of association for those HLA alleles that can be predicted from the set of SNPs described in Methods at an $r^{2}>0.80$ (47 out of the 70 non-DRB1 HLA alleles, or 67\%), and that moreover show conditional association at the level $p<0.001$. Table 1 shows, for each HLA allele, its frequency as estimated from the data from de Bakker et al. [8], the SNP or the combination of SNPs that can be used to predict the HLA allele, along with the predictor allele or haplotype, and the strength of the prediction in terms of the $r^{2}$ measure of LD. It also shows the results of the conditional tests of association, including the conditional ORs and their confidence intervals.

We find that two class II alleles, DQA1*0301 and DQB1*0302, and one class I allele, $\mathrm{B}^{*} 0801$, show significant association with RA irrespective of the presence or absence, on their respective haplotypes, of an SE allele at the DRB1 locus. For DQA1*0301, both conditional ORs are estimated to take the same value, 
Table I: Conditional tests of association between RA and classical HLA alleles through SNP surrogates

\begin{tabular}{|c|c|c|c|c|c|c|c|c|c|c|}
\hline \multirow[b]{2}{*}{ HLA allele } & \multirow[b]{2}{*}{ Frequency $^{a}$} & \multirow[b]{2}{*}{ Surrogate (allele/haplotype) ${ }^{\text {b }}$} & \multirow[b]{2}{*}{$r^{2 c}$} & \multirow[b]{2}{*}{$p$-value ${ }^{d}$} & \multicolumn{3}{|c|}{ SE- ${ }^{-}$} & \multicolumn{3}{|c|}{$S E+{ }^{f}$} \\
\hline & & & & & OR & Cl-low & Cl-high & OR & Cl-low & Cl-high \\
\hline DQA*030I & 0.27 & rs660895 (G) & 0.96 & $2.13 \times 10^{-12}$ & 2.11 & 1.38 & 3.24 & 2.11 & 1.65 & 2.71 \\
\hline$D Q B * 0501$ & 0.11 & rs17533090, rs9275406, rs9275439 (AAG) & 1.00 & $4.30 \times 10^{-11}$ & 0.33 & 0.04 & 2.61 & 0.43 & 0.33 & 0.55 \\
\hline DQA*0I0I & 0.13 & rs9268832, rs2395।85, rs7774434 (GCG)' & 0.80 & $5.12 \times 10^{-08}$ & 0.58 & 0.20 & 1.68 & 0.48 & 0.37 & 0.62 \\
\hline$B * 0801$ & 0.16 & rs3134792 (C) & 1.00 & $1.86 \times 10^{-4}$ & 1.54 & 1.03 & 2.30 & 3.03 & 1.27 & 7.23 \\
\hline DQB*0302 & 0.19 & rs92753I2 (G) & 0.94 & $1.96 \times 10^{-4}$ & 2.23 & 1.28 & 3.89 & 1.38 & 1.07 & 1.79 \\
\hline$C^{*} * 401$ & 0.08 & rs9264904 (A) & 1.00 & $1.99 \times 10^{-4}$ & 0.85 & 0.56 & 1.30 & 0.59 & 0.44 & 0.79 \\
\hline
\end{tabular}

${ }^{\mathrm{a}}$ Frequency estimated from the data of de Bakker et al. [8].

${ }^{\mathrm{b}}$ Alleles or haplotypes used as surrogate for the HLA alleles, with corresponding SNPs.

$c^{2} r^{2}$ measure of linkage disequilibrium between the HLA allele and the surrogate.

${ }^{d} p$-value for the two degrees of freedom conditional test of association.

e Odds ratio and $95 \%$ confidence interval for HLA alleles on non-SE allele bearing haplotypes.

${ }^{f}$ Odds ratio and $95 \%$ confidence interval for HLA alleles on SE allele bearing haplotypes.

$2.11\left(p=2 \times 10^{-12}\right)$. For DQB1*0302, the risk is higher when not combined with an SE allele (2.23 versus 1.38 , $p=0.0002)$. The alpha and beta chains DQA1*0301/ $\mathrm{DQB1}{ }^{*} 0302$ together form the DQ8 serotype [13]. That they are co-associated is thus not surprising. DQ8 has been shown to be associated with RA in humans [14], but this association was thought to be due to LD with DRB $1 * 0401$ and $* 0405$ (two SE alleles). Our results are indicative of DQ8 being a risk factor independent of the risk alleles, or non-risk alleles, found at DRB1 (but see Discussion). For the class I allele B*0801, the OR is 1.54 when its haplotype does not contain an SE allele, while it is 3.03 otherwise $(p=0.00018)$. $\mathrm{B}^{*} 0801$ is found on the ancestral 8.1 haplotype, which has been shown to carry risk for RA as well as DRB1*03, a non-SE allele [15]. All other HLA alleles from Table 1 show significant decrease in risk only when combined with an SE allele (see Discussion).

\section{Discussion}

Conditioning on the presence or absence of SE alleles on the same haplotype as the test allele at other HLA loci helps reduce the confounding effects of LD with the DRB1 locus, but since different DRB1 alleles, or combinations thereof, show a wide spectrum of risks, this conditioning argument is not sufficient on its own to fully account for DRB1. Knowledge of the haplotype structure in the MHC region is still necessary for a better interpretation of the results. For instance, the apparent protection that seems to be conferred by DQB1*0501 or DQA $1 * 0101$ (Table 1 ) is a mere reflection of the fact that these two alleles are in LD with DR1/DR10 [16], which although they are risk factors for RA, they are not the most prominent ones [14]. Moreover, the classical HLA alleles that we report in the present study are only predicted from the SNP data at hand, sometimes imperfectly, and based on only a small sample (in our case, the HapMap CEU samples). Thus, it is still unclear if the associations seen between DQA1*0301/ DQB1*0302 (the DQ8 serotype) and RA truly reflect risks that are independent of DRB1, or rather are artifacts of the measurement errors inherent to any tagging procedure. In terms of the power to detect diseaseassociated HLA alleles, a penalty is incurred when using SNPs or SNP-haplotypes as surrogates for them, because the sample size required to achieve a given power is inversely proportional to the $r^{2}$ measure of LD between them [17]. Yet, as a proof-of-concept and justification for the more expensive typing of HLA alleles at high resolution, using SNP data and publicly available databases of HLA data to predict classical class I and class II alleles is an efficient method for preliminary evaluation of the role of HLA genes in the etiology of autoimmune, infectious or other relevant diseases.

\section{List of abbreviations used}

HLA: Human leukocyte antigen; LD: Linkage disequilibrium; MHC: Major histocompatibility complex; NARAC: North American Rheumatoid Arthritis Consortium; OR: Odds ratio; RA: Rheumatoid arthritis; SE: Shared epitope; SNP: Single-nucleotide polymorphisms.

\section{Competing interests}

The author declares that he has no competing interests.

\section{Acknowledgements}

The author thanks Nicole Roslin and two anonymous reviewers for their comments on earlier versions of this manuscript. The Genetic Analysis Workshops are supported by NIH grant ROI GM03I575 from the National Institute of General Medical Sciences.

This article has been published as part of BMC Proceedings Volume 3 Supplement 7, 2009: Genetic Analysis Workshop 16. The full contents of the supplement are available online at http://www.biomedcentral.com/ $\underline{1753-656 \mid / 3 \text { ? issue }=S 7 .}$. 


\section{References}

I. Newton JL, Harney SM, Wordsworth BP and Brown MA: A review of the MHC genetics of rheumatoid arthritis. Genes Immun 2004, 5: $15 \mid-157$

2. Plenge RM, Cotsapas C, Davies L, Price AL, de Bakker PI, Maller J, Pe'er I, Burtt NP, Blumenstiel B, DeFelice M, Parkin M, Barry R, Winslow W, Healy C, Graham RR, Neale BM, Izmailova E, Roubenoff R, Parker AN, Glass R, Karlson EW, Maher N, Hafler DA, Lee DM, Seldin MF, Remmers EF, Lee AT, Padyukov L, Alfredsson L, Coblyn J, Weinblatt ME, Gabriel SB, Purcell S, Klareskog L, Gregersen PK, Shadick NA, Daly MJ and Altshuler D: Two independent alleles at $6 \mathrm{q} 23$ associated with risk of rheumatoid arthritis. Nat Genet 2007, 39:|477-|482.

3. Plenge RM, Seielstad M, Padyukov L, Lee AT, Remmers EF, Ding B, Liew A, Khalili H, Chandrasekaran A, Davies LR, Li W, Tan AK, Bonnard C, Ong RT, Thalamuthu A, Pettersson S, Liu C, Tian C, Chen WV, Carulli JP, Beckman EM, Altshuler D, Alfredsson L, Criswell LA, Amos Cl, Seldin MF, Kastner DL, Klareskog L and Gregersen PK: TRAFI-C5 as a risk locus for rheumatoid arthritis-a genomewide study. N Engl J Med 2007, 357:1199-1209.

4. Wellcome Trust Case Control Consortium: Genome-wide association study of I4,000 cases of seven common diseases and 3,000 shared controls. Nature 2007, 447:66I-678.

5. Begovich $A B$, Carlton VE, Honigberg LA, Schrodi SJ, Chokkalingam AP, Alexander HC, Ardlie KG, Huang Q, Smith AM, Spoerke JM, Conn MT, Chang M, Chang SY, Saiki RK, Catanese J], Leong DU, Garcia VE, McAllister LB, Jeffery DA, Lee AT, Batliwalla F, Remmers E, Criswell LA, Seldin MF, Kastner DL, Amos CI, Sninsky JJ and Gregersen PK: A missense single-nucleotide polymorphism in a gene encoding a protein tyrosine phosphatase (PTPN22) is associated with rheumatoid arthritis. Am J Hum Genet 2004, 75:330-337.

6. Gregersen PK, Silver J and Winchester RJ: The shared epitope hypothesis. An approach to understanding the molecular genetics of susceptibility to rheumatoid arthritis. Arthritis Rheum 1987, 30:1205-1213.

7. du Montcel ST, Michou L, Petit-Teixeira E, Osorio J, Lemaire I, Lasbleiz S, Pierlot C, Quillet P, Bardin T, Prum B, Cornelis F and Clerget-Darpoux F: New classification of HLA-DRB I alleles supports the shared epitope hypothesis of rheumatoid arthritis susceptibility. Arthritis Rheum 2005, 52:1063-1068.

8. de Bakker PI, McVean G, Sabeti PC, Miretti MM, Green T, Marchini J, Ke X, Monsuur AJ, Whittaker $P$, Delgado M, Morrison J, Richardson A, Walsh EC, Gao X, Galver L, Hart J, Hafler DA, Pericak-Vance M, Todd JA, Daly MJ, Trowsdale J, Wijmenga C, Vyse TJ, Beck S, Murray SS, Carrington M, Gregory S, Deloukas P and Rioux JD: A high-resolution HLA and SNP haplotype map for disease association studies in the extended human MHC. Nat Genet 2006, 38: I I66-I I72.

9. de Bakker PI, Yelensky R, Pe'er I, Gabriel SB, Daly MJ and Altshuler D: Efficiency and power in genetic association studies. Nat Genet 2005, 37:1217-1223.

10. Barrett JC, Fry B, Maller J and Daly MJ: Haploview: analysis and visualization of LD and haplotype maps. Bioinformatics 2005, 2I:263-265

II. Dudbridge F: Likelihood-based association analysis for nuclear families and unrelated subjects with missing genotype data. Hum Hered 2008, 66:87-98.

12. Price AL, Patterson NJ, Plenge RM, Weinblatt ME, Shadick NA and Reich D: Principal components analysis corrects for stratification in genome-wide association studies. Nat Genet 2006, 38:904-909.

13. Perdriger A: Do the HLA-DO and DP genes play a role in rheumatoid arthritis?. Joint Bone Spine 200I, 68:12-I8.

14. de Vries N, van Elderen C, Tijssen H, van Riel PL and Putte van de LB: No support for HLA-DQ encoded susceptibility in rheumatoid arthritis. Arthritis Rheum 1999, 42: |62I-1627.

15. Jawaheer D, Li W, Graham RR, Chen W, Damle A, Xiao X, Monteiro I, Khalili $H$, Lee A, Lundsten R, Begovich A, Bugawan $T$, Erlich H, Elder JT, Criswell LA, Seldin MF, Amos Cl, Behrens TW and Gregersen PK: Dissecting the genetic complexity of the association between human leukocyte antigens and rheumatoid arthritis. Am J Hum Genet 2002, 7l:585-594.

16. Vos K, Horst-Bruinsma van der IE, Hazes JM, Breedveld FC, le Cessie S, Schreuder GM, de Vries RR and Zanelli E: Evidence for a protective role of the human leukocyte antigen class II region in early rheumatoid arthritis. Rheumatology 200I, 40:133-139.
17. Wang WY, Barratt BJ, Clayton DG and Todd JA: Genome-wide association studies: theoretical and practical concerns. Nat Rev Genet 2005, 6:109-II8.
Publish with Biomed Central and every scientist can read your work free of charge

"BioMed Central will be the most significant development for disseminating the results of biomedical research in our lifetime. "

Sir Paul Nurse, Cancer Research UK

Your research papers will be:

- available free of charge to the entire biomedical community

- peer reviewed and published immediately upon acceptance

- cited in PubMed and archived on PubMed Central

- yours - you keep the copyright 